\title{
Quark number fluctuations in the Polyakov loop-extended quark-meson model at finite baryon density
}

\author{
V. Skokov, ${ }^{1, *}$ B. Friman, ${ }^{1}$ and K. Redlich ${ }^{2,3}$ \\ ${ }^{1}$ GSI Helmholtzzentrum für Schwerionenforschung, DE-64291 Darmstadt, Germany \\ ${ }^{2}$ Institute of Theoretical Physics, University of Wroclaw, PL-50204 Wrocław, Poland \\ ${ }^{3}$ Theory Division, CERN, CH-1211 Geneva 23, Switzerland \\ (Received 26 February 2011; published 12 May 2011)
}

\begin{abstract}
The thermodynamics and phase structure of the Polyakov loop-extended two-flavor chiral quark-meson (PQM) model are explored beyond the mean-field approximation. The analysis of the PQM model is based on the functional renormalization group (FRG) method. We formulate and solve the renormalization group flow equation for the scale-dependent thermodynamic potential in the presence of the gluonic background field at finite temperature and density. We determine the phase diagram of the PQM model in the FRG approach and discuss its modification compared to the mean-field approximation. We focus on the fluctuations of the net-quark number density, including higher cumulants, and discuss the influence of nonperturbative effects near the chiral crossover transition. We find that, with increasing net-quark number density, the higher-order cumulants exhibit a characteristic structure near the transition. We also consider ratios of different cumulants of the net-quark number density and discuss their role as probes of the deconfinement and chiral phase transitions.
\end{abstract}

DOI: 10.1103/PhysRevC.83.054904

PACS number(s): 24.85.+p, 21.65.-f, 25.75.-q, 24.60.-k

\section{INTRODUCTION}

Thermodynamic properties of strongly interacting matter at nonzero baryon density and high temperature have been explored numerically within lattice quantum chromodynamics (LQCD) [1-3]. The LQCD results show that QCD exhibits both restoration of chiral symmetry and deconfinement at finite temperatures and densities. The LQCD equation of state indicates a clear separation between the confined hadronic and the deconfined quark-gluon plasma phases. However, the thermodynamics of strongly interacting matter at large baryon densities and for small quark masses is presently not accessible in first-principles LQCD calculations. Here, phenomenological models and effective theories offer a viable framework for exploratory studies [4-18].

The properties of low-energy hadrons as well as the nature of the chiral phase transition at finite temperature and density have been studied intensively in such effective models. Recently, the physics of color confinement and the relation to chiral symmetry breaking also have been addressed in a similar framework. The idea to extend the chiral Lagrangians, such as the Nambu-Jona-Lasinio or the quark-meson models, by introducing a coupling of quarks to a uniform temporal background of gauge fields (the Polyakov loop) was an important step forward in these investigations [7,15].

The Polyakov loop-extended Nambu-Jona-Lasinio (PNJL) [9] and quark-meson (PQM) [15] models reproduce essential features of the QCD thermodynamics already in the mean-field approximation. However, to correctly account for the critical behavior and scaling properties near the chiral phase transition, it is necessary to go beyond the mean-field approximation and include fluctuations and nonperturbative dynamics. This can

\footnotetext{
*v.skokov@gsi.de
}

be achieved, e.g., by using methods based on the functional renormalization group (FRG) [19-25].

To account for fluctuations in the thermodynamics of the PQM model, we solve the suitably truncated FRG flow equations for fluctuations of the meson fields, while the Polyakov loop is treated as a background field on the meanfield level. We extend our previous work [26] to nonzero chemical potential and locate the phase boundary and the critical end point (CEP) by exploring the dependence of the chiral order parameter and the quark number susceptibility on the thermodynamic variables.

The cumulants of the net-quark number density $\left(c_{n}\right)$ are computed at finite temperature and chemical potential, including the effects of mesonic fluctuations. We also discuss the role of nonperturbative effects on the properties of the first four cumulants near the chiral crossover transition. In particular, we show that the cumulants exhibit a characteristic structure and turn negative in the vicinity of the transition for sufficiently large values of the chemical potential. Furthermore, the role of the ratios $c_{3} / c_{1}$ and $c_{4} / c_{2}$ as probes of the deconfinement and chiral phase transitions is discussed. Finally, we summarize the results for the various susceptibilities near the chiral phase transition at finite net-quark density in the Landau (mean-field) and scaling theories.

\section{THE POLYAKOV QUARK-MESON MODEL}

The quark-meson model is an effective realization of the low-energy sector of QCD, which incorporates chiral symmetry. However, because the local color $\mathrm{SU}\left(N_{c}\right)$ invariance of QCD is replaced by a global symmetry, the model does not describe confinement. Nevertheless, by introducing a coupling of the quarks to a uniform temporal color gauge field, represented by the Polyakov loop, many facets of confinement can be emulated $[7,14,15]$. 
The Lagrangian of the PQM model reads as [15]

$$
\begin{aligned}
\mathcal{L}= & \bar{q}\left[i \gamma^{\mu} D_{\mu}-g\left(\sigma+i \gamma_{5} \vec{\tau} \vec{\pi}\right)\right] q+\frac{1}{2}\left(\partial_{\mu} \sigma\right)^{2}+\frac{1}{2}\left(\partial_{\mu} \vec{\pi}\right)^{2} \\
& -U(\sigma, \vec{\pi})-\mathcal{U}\left(\ell, \ell^{*}\right) .
\end{aligned}
$$

The coupling between the effective gluon field and quarks is implemented through the covariant derivative

$$
D_{\mu}=\partial_{\mu}-i A_{\mu},
$$

where $A_{\mu}=g A_{\mu}^{a} \lambda^{a} / 2$. The spatial components of the gluon field are neglected, i.e., $A_{\mu}=\delta_{\mu 0} A_{0}$. Moreover, $\mathcal{U}\left(\ell, \ell^{*}\right)$ is the effective potential for the gluon field expressed in terms of the thermal expectation values of the color trace of the Polyakov loop and its conjugate

$$
\ell=\frac{1}{N_{c}}\left\langle\operatorname{Tr}_{c} L(\vec{x})\right\rangle, \quad \ell^{*}=\frac{1}{N_{c}}\left\langle\operatorname{Tr}_{c} L^{\dagger}(\vec{x})\right\rangle,
$$

with

$$
L(\vec{x})=\mathcal{P} \exp \left[i \int_{0}^{\beta} d \tau A_{4}(\vec{x}, \tau)\right],
$$

where $\mathcal{P}$ stands for the path ordering, $\beta=1 / T$, and $A_{4}=$ $i A_{0}$. In the $O(4)$ representation, the meson field is introduced as $\phi=(\sigma, \vec{\pi})$ and the corresponding $\mathrm{SU}(2)_{L} \otimes \mathrm{SU}(2)_{R}$ chiral representation is defined by $\sigma+i \vec{\tau} \cdot \vec{\pi} \gamma_{5}$.

The purely mesonic potential of the model $U(\sigma, \vec{\pi})$ is defined as

$$
U(\sigma, \vec{\pi})=\frac{\lambda}{4}\left(\sigma^{2}+\vec{\pi}^{2}-v^{2}\right)^{2}-c \sigma,
$$

while the effective potential of the gluon field is parametrized in such a way as to preserve the $Z(3)$ invariance:

$$
\frac{\mathcal{U}\left(\ell, \ell^{*}\right)}{T^{4}}=-\frac{b_{2}(T)}{2} \ell^{*} \ell-\frac{b_{3}}{6}\left(\ell^{3}+\ell^{* 3}\right)+\frac{b_{4}}{4}\left(\ell^{*} \ell\right)^{2} .
$$

The parameters

$$
b_{2}(T)=a_{0}+a_{1}\left(\frac{T_{0}}{T}\right)+a_{2}\left(\frac{T_{0}}{T}\right)^{2}+a_{3}\left(\frac{T_{0}}{T}\right)^{3}
$$

with $a_{0}=6.75, a_{1}=-1.95, a_{2}=2.625, a_{3}=-7.44, b_{3}=$ $0.75, b_{4}=7.5$, and $T_{0}=270 \mathrm{MeV}$ were chosen to reproduce the equation of state of the pure $\mathrm{SU}_{c}(3)$ lattice gauge theory. When the coupling to the quark degrees of freedom is neglected, the potential (6) yields a first-order deconfinement phase transition at $T_{0}$. Several alternative parametrizations of the effective potential of the gluon field were explored in Refs. [27-29].

\section{A. The FRG method in the PQM model}

To take fluctuations into account in the PQM model, we employ a scheme based on the functional renormalization group (FRG). This scheme involves an infrared regularization of the fluctuations at a sliding momentum scale $k$, resulting in a scale-dependent effective action $\Gamma_{k}$, the so-called effective average action [19-22]. We treat the Polyakov loop as a background field, which is introduced self-consistently on the mean-field level, while for the quark and meson fields, fluctuations are accounted for by solving the FRG flow equations.

The evolution of $\Gamma_{k}$ with the changing momentum scale is then described by the flow equation

$$
\begin{aligned}
\partial_{k} \Gamma_{k}[\Phi, \psi]= & \frac{1}{2} \operatorname{Tr}\left\{\partial_{k} R_{k B}\left(\Gamma_{k}^{(2,0)}[\Phi, \psi]+R_{k B}\right)^{-1}\right\} \\
& -\operatorname{Tr}\left\{\partial_{k} R_{k F}\left(\Gamma_{k}^{(0,2)}[\Phi, \psi]+R_{k F}\right)^{-1}\right\},
\end{aligned}
$$

where $\Gamma_{k}^{(2,0)}$ and $\Gamma_{k}^{(0,2)}$ denote the second functional derivative of $\Gamma_{k}[\Phi, \psi]$ with respect to the bosonic $(\Phi)$ and fermionic $(\psi)$ fields, respectively. These derivatives correspond to the inverse of the full propagators at the scale $k$. The trace in Eq. (8) denotes a momentum integration as well as a summation over Matsubara frequencies and all internal indices. Following our previous work [26], we formulate the flow equation for the scale-dependent grand canonical potential density $\Omega_{k}=T \Gamma_{k} / V$ for the quark and meson subsystems

$$
\begin{aligned}
\partial_{k} \Omega_{k}\left(\ell, \ell^{*} ; T, \mu\right) \\
=\frac{k^{4}}{12 \pi^{2}}\left\{\frac{3}{E_{\pi}}\left[1+2 n_{B}\left(E_{\pi} ; T\right)\right]+\frac{1}{E_{\sigma}}\left[1+2 n_{B}\left(E_{\sigma} ; T\right)\right]\right. \\
\left.\quad-\frac{4 N_{c} N_{f}}{E_{q}}\left[1-N\left(\ell, \ell^{*} ; T, \mu\right)-\bar{N}\left(\ell, \ell^{*} ; T, \mu\right)\right]\right\} .
\end{aligned}
$$

Here $n_{B}\left(E_{\pi, \sigma} ; T\right)$ is the bosonic distribution function

$$
n_{B}\left(E_{\pi, \sigma} ; T\right)=\frac{1}{\exp \left(E_{\pi, \sigma} / T\right)-1},
$$

with the pion and sigma energies

$$
E_{\pi}=\sqrt{k^{2}+\bar{\Omega}_{k}^{\prime}}, \quad E_{\sigma}=\sqrt{k^{2}+\bar{\Omega}_{k}^{\prime}+2 \rho \bar{\Omega}_{k}^{\prime \prime}},
$$

where the primes denote derivatives with respect to $\rho=\left(\sigma^{2}+\right.$ $\left.\vec{\pi}^{2}\right) / 2$ of $\bar{\Omega}=\Omega+c \sigma$. The fermion distribution functions $N\left(\ell, \ell^{*} ; T, \mu\right)$ and $\bar{N}\left(\ell, \ell^{*} ; T, \mu\right)$,

$$
\begin{aligned}
& N\left(\ell, \ell^{*} ; T, \mu\right)=\frac{1+2 \ell^{*} \exp \left[\beta\left(E_{q}-\mu\right)\right]+\ell \exp \left[2 \beta\left(E_{q}-\mu\right)\right]}{1+3 \ell \exp \left[2 \beta\left(E_{q}-\mu\right)\right]+3 \ell^{*} \exp \left[\beta\left(E_{q}-\mu\right)\right]+\exp \left[3 \beta\left(E_{q}-\mu\right)\right]}, \\
& \bar{N}\left(\ell, \ell^{*} ; T, \mu\right)=N\left(\ell^{*}, \ell ; T,-\mu\right)
\end{aligned}
$$

are modified because of the coupling to the gluon field. Finally, the quark energy is given by

$$
E_{q}=\sqrt{k^{2}+2 g^{2} \rho} .
$$

The minimum of the thermodynamic potential is determined by the stationarity condition

$$
\left.\frac{d \Omega_{k}}{d \sigma}\right|_{\sigma=\sigma_{k}}=\left.\frac{d \bar{\Omega}_{k}}{d \sigma}\right|_{\sigma=\sigma_{k}}-c=0 .
$$


The flow Eq. (9) is solved numerically with the initial cutoff $\Lambda=1.2 \mathrm{GeV}$ (see details in Ref. [26]). The initial conditions for the flow are chosen to reproduce the in-vacuum properties: the physical pion mass $m_{\pi}=138 \mathrm{MeV}$, the pion decay constant $f_{\pi}=93 \mathrm{MeV}$, the sigma mass $m_{\sigma}=600 \mathrm{MeV}$, and the constituent quark mass $m_{q}=300 \mathrm{MeV}$ at the scale $k \rightarrow 0$. The symmetry-breaking term $c=m_{\pi}^{2} f_{\pi}$ corresponds to an external field and consequently does not flow. In this paper, we neglect the flow of the Yukawa coupling $g$, which is not expected to be significant for these studies (see, e.g., Refs. [30,31]).

By solving Eq. (9), one obtains the thermodynamic potential for the quark and mesonic subsystems $\Omega_{k \rightarrow 0}\left(\ell, \ell^{*} ; T, \mu\right)$ as a function of the Polyakov loop variables $\ell$ and $\ell^{*}$. The full thermodynamic potential $\Omega\left(\ell, \ell^{*} ; T, \mu\right)$ in the PQM model, including quark, meson, and gluon degrees of freedom, is obtained by adding the effective gluon potential $\mathcal{U}\left(\ell, \ell^{*}\right)$ to $\Omega_{k \rightarrow 0}\left(\ell, \ell^{*} ; T, \mu\right)$ :

$$
\Omega\left(\ell, \ell^{*} ; T, \mu\right)=\Omega_{k \rightarrow 0}\left(\ell, \ell^{*} ; T, \mu\right)+\mathcal{U}\left(\ell, \ell^{*}\right) .
$$

At a given temperature and chemical potential, the Polyakov loop variables $\ell$ and $\ell^{*}$ are then determined by the stationarity conditions

$$
\begin{aligned}
& \frac{\partial}{\partial \ell} \Omega\left(\ell, \ell^{*} ; T, \mu\right)=0, \\
& \frac{\partial}{\partial \ell^{*}} \Omega\left(\ell, \ell^{*} ; T, \mu\right)=0 .
\end{aligned}
$$

The thermodynamic potential (14) does not contain contributions of thermal modes with momenta larger than the cutoff $\Lambda$. To obtain the correct high-temperature behavior of thermodynamic functions, we need to supplement the FRG potential with the contribution of the high-momentum states. A simplified model for implementing such states was proposed in Ref. [32], where the contributions of the $k>\Lambda$ states to the flow are approximated by those of a noninteracting gas of quarks and gluons. For the PQM model, this procedure was generalized in Ref. [26] by including the flow of quarks interacting with the Polyakov loop for $k>\Lambda$ :

$$
\begin{aligned}
\partial_{k} & \Omega_{k}^{\Lambda}(T, \mu) \\
& =-\frac{N_{c} N_{f} k^{3}}{3 \pi^{2}}\left[1-N\left(\ell, \ell^{*} ; T, \mu\right)-\bar{N}\left(\ell, \ell^{*} ; T, \mu\right)\right] .
\end{aligned}
$$

Here, the dynamical quark mass is small compared to the momentum $k$ and consequently neglected. Moreover, since the effective gluon potential $\mathcal{U}\left(\ell, \ell^{*}\right)$ is fitted to reproduce the gluonic Stefan-Boltzmann limit at high temperatures, the explicit gluon contribution is, for consistency, not included in (17).

To obtain the complete thermodynamic potential of the PQM model, we thus integrate Eq. (17) from $k=\infty$ to $k=\Lambda$, where we switch to the PQM flow Eq. (9). The first term in the high-momentum flow Eq. (17) diverges in the ultraviolet. However, since it is independent of mesonic as well as gluonic fields, temperature, and chemical potential, the divergent contribution can be absorbed in an unobservable constant shift of the thermodynamic potential.

\section{B. The mean-field approximation}

To illustrate the importance of mesonic fluctuations on the thermodynamics of the PQM model, we compare the FRG results with those obtained in the mean-field approximation. In the latter, mesonic fluctuations are neglected and the mesonic fields are replaced by their classical expectation values.

In the PQM model, the thermodynamical potential in the mean-field approximation reads as [15]

$$
\Omega_{\mathrm{MF}}=\mathcal{U}\left(\ell, \ell^{*}\right)+U(\langle\sigma\rangle,\langle\pi\rangle=0)+\Omega_{q \bar{q}}\left(\langle\sigma\rangle, \ell, \ell^{*}\right) .
$$

Here, the contribution of quarks with the dynamical mass $m_{q}=$ $g\langle\sigma\rangle$ is given by

$$
\begin{aligned}
\Omega_{q \bar{q}}\left(\langle\sigma\rangle, \ell, \ell^{*}\right) \\
=-2 N_{f} T \int \frac{d^{3} p}{(2 \pi)^{3}}\left\{\frac{N_{c} E_{q}}{T}+\ln g^{(+)}\left(\langle\sigma\rangle, \ell, \ell^{*} ; T, \mu\right)\right. \\
\left.\quad+\ln g^{(-)}\left(\langle\sigma\rangle, \ell, \ell^{*} ; T, \mu\right)\right\},
\end{aligned}
$$

where

$$
\begin{aligned}
g^{(+)}\left(\langle\sigma\rangle, \ell, \ell^{*} ; T, \mu\right)= & 1+3 \ell \exp \left[-\left(E_{q}-\mu\right) / T\right] \\
& +3 \ell^{*} \exp \left[-2\left(E_{q}-\mu\right) / T\right] \\
& +\exp \left[-3\left(E_{q}-\mu\right) / T\right], \\
g^{(-)}\left(\langle\sigma\rangle, \ell, \ell^{*} ; T, \mu\right)= & g^{(+)}\left(\langle\sigma\rangle, \ell^{*}, \ell ; T,-\mu\right)
\end{aligned}
$$

and $E_{q}=\sqrt{p^{2}+m_{q}^{2}}$ is the quark quasiparticle energy. The first term in Eq. (19) is a divergent vacuum fluctuation contribution, which has to be properly regularized. Following Ref. [33], we employ dimensional regularization. The finite contribution of the vacuum term to Eq. (19) reads as [33]

$$
\Omega_{q \bar{q}}^{\mathrm{vac}}=-\frac{N_{c} N_{f}}{8 \pi^{2}} m_{q}^{4} \ln \left(\frac{m_{q}}{M}\right),
$$

where $M$ is the renormalization scale; the resulting thermodynamic potential $\Omega_{\mathrm{MF}}$ is independent of $M$. The relevance of the vacuum contribution for the thermodynamics of chiral models was demonstrated and studied in detail in Refs. [33] and [34].

The equations of motion for the mean fields are obtained by requiring that the thermodynamic potential is stationary with respect to changes of $\langle\sigma\rangle, \ell$, and $\ell^{*}$ :

$$
\frac{\partial \Omega_{\mathrm{MF}}}{\partial\langle\sigma\rangle}=\frac{\partial \Omega_{\mathrm{MF}}}{\partial \ell}=\frac{\partial \Omega_{\mathrm{MF}}}{\partial \ell^{*}}=0 .
$$

The model parameters are fixed to reproduce the same vacuum physics as in the FRG calculation.

\section{THERMODYNAMICS OF THE PQM MODEL}

We now explore the critical properties of the PQM model at finite baryon density using the functional renormalization group and in the mean-field approximation. In the FRG 
approach, the thermodynamic potential (14) at finite temperature and chemical potential is obtained by solving the flow Eq. (9) numerically, using the Taylor expansion method [26,35]. This approach has been successfully applied to the thermodynamics at finite density and temperature [24,26,34] in the regime where the system exhibits a crossover or a secondorder phase transition. In the regime of a first-order phase transition, where the thermodynamical potential develops two degenerate minima, a different numerical method is needed for solving the FRG flow equations [34,36]. In this paper, we restrict our considerations to the parameter range where the PQM model exhibits a crossover or a second-order chiral phase transition.

\section{A. The phase diagram}

The PQM model, which is expected to belong to the same universality class as QCD, should, for a range of parameters, exhibit a generic phase diagram with a critical point at nonvanishing chemical potential. In the chiral limit, a second-order phase boundary is identified by a singularity of the chiral susceptibility. For finite quark masses, the chiral transition at small values of the chemical potential is of the crossover type. In this case, the pseudocritical temperature and chemical potential are determined by locating the maximum of the chiral susceptibility or of the temperature derivative of the chiral order parameter. Furthermore, the position of the CEP is revealed by a zero of the sigma-meson mass, or equivalently by a divergence of the net quark-number susceptibility.

In Fig. 1, we show the phase diagram of the PQM model obtained in the FRG approach and in the mean-field approximation. For a physical pion mass and moderate values of the chemical potential, the PQM model exhibits a smooth crossover chiral transition. In Fig. 1, the transition region is shown as a band where the temperature derivative of the order parameter is above $95 \%$ of its maximal value. At larger values of the chemical potential, the crossover line terminates at the CEP, where the transition is second order, belonging to the universality class of the three-dimensional Ising model.

In the following, we focus on observables that are related to the net-quark number density and consider cumulants of the quark number fluctuations. We discuss the dependence of these fluctuations on the quark chemical potential within the FRG approach in the presence of the gluonic background.

\section{B. Quark number density fluctuations}

Fluctuations of conserved charges provide direct information on the critical properties related to chiral symmetry restoration. The fluctuations of the baryon number are of particular interest. In equilibrium, a divergence of the netquark number susceptibility is connected with the existence of the CEP [37]. Consequently, a nonmonotonic dependence of these fluctuations on collision energy in heavy-ion collisions was proposed as a signature for the CEP [37]. A diverging net-quark number susceptibility also signals the spinodal decomposition of a nonequlibrium first-order chiral phase transition [10].

The fluctuations of the net-quark number are characterized by the generalized susceptibilities

$$
c_{n}(T)=\frac{\partial^{n}\left[p(T, \mu) / T^{4}\right]}{\partial(\mu / T)^{n}} .
$$

The first coefficient $c_{1}=n_{q} / T^{3}$ characterizes the response of the net-quark number to changes in the quark chemical potential, while the second coefficient

$$
c_{2}=\frac{\chi_{q}}{T^{2}}=\frac{1}{V T^{3}}\left\langle\left(\delta N_{q}\right)^{2}\right\rangle,
$$

with $\delta N_{q}=N_{q}-\left\langle N_{q}\right\rangle$, is proportional to the susceptibility of the net-quark number density $\chi_{q}$. The third- and fourth-order cumulants can be expressed through the fluctuation of the net-quark number $\delta N_{q}$ :

$$
\begin{aligned}
c_{3} & =\frac{1}{V T^{3}}\left\langle\left(\delta N_{q}\right)^{3}\right\rangle, \\
c_{4} & =\frac{1}{V T^{3}}\left[\left\langle\left(\delta N_{q}\right)^{4}\right\rangle-3\left\langle\left(\delta N_{q}\right)^{2}\right\rangle^{2}\right] .
\end{aligned}
$$

The coefficients $c_{n}(T)$ are sensitive probes of the chiral phase transition. They indicate the position, the order, and, in case of the second-order phase transition, the universality class of the corresponding phase transition. The net-quark number
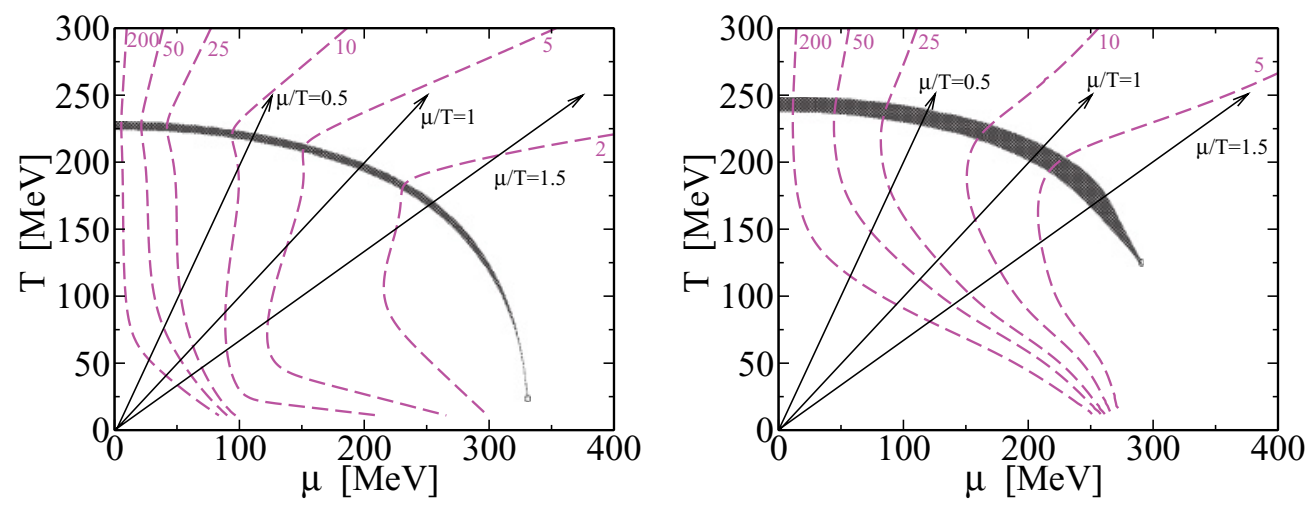

FIG. 1. (Color online) The phase diagrams for the PQM model in the mean-field approximation (left panel) and in the FRG approach (right panel). In the shaded regions, the temperature derivative of the chiral order parameter $d \sigma / d T$ is above $95 \%$ of its maximal value. The arrows are lines of constant $\mu / T$ and the dashed curves show isentropes for $s / n_{q}=2,5,10,25,50$, and 200. 

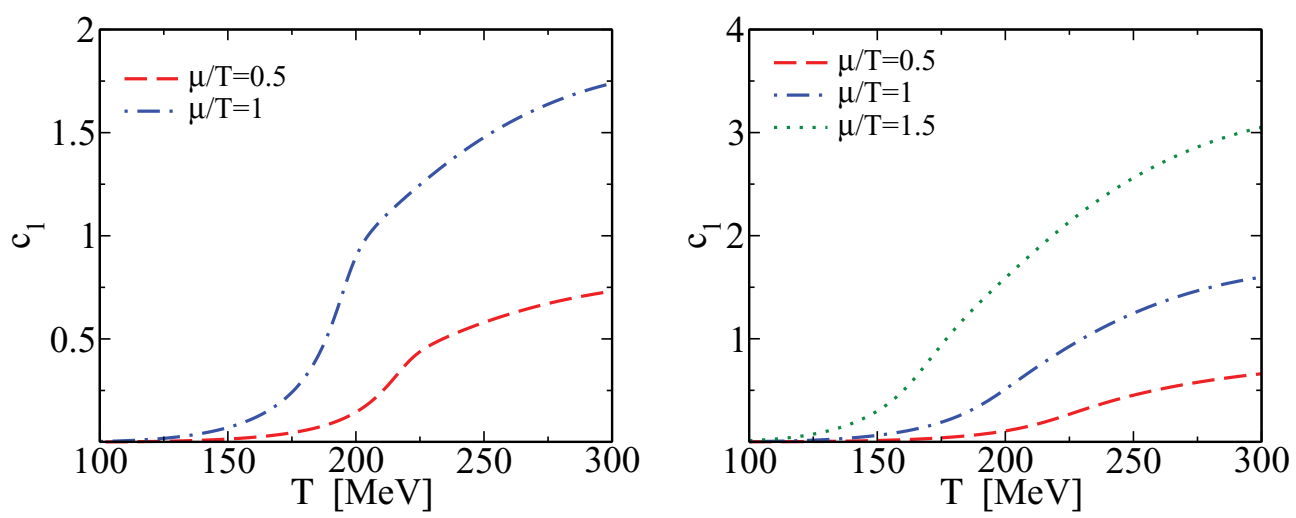

FIG. 2. (Color online) The quark number density normalized by $T^{3}, c_{1}=n_{q} / T^{3}$, as a function of temperature for different values of $\mu / T$ for the PQM model in the mean-field approximation (left panel) and in the FRG approach (right panel).

density $n_{q}$ is discontinuous at a first-order transition, whereas the susceptibility $c_{2}$ and higher cumulants diverge at the CEP [38-40]. In the chiral limit and at nonzero chemical potential, all generalized susceptibilities $c_{n}(T)$ with $n>2$ diverge at the $O(4)$ chiral critical line [41]. Moreover, they also diverge at the spinodal lines of a first-order chiral phase transition [10].

A particular role is attributed to the so-called kurtosis of the net-quark number fluctuations [41-43], which is defined as the ratio

$$
R_{4,2}=\frac{c_{4}}{c_{2}} .
$$

This key observable reflects both the chiral and the deconfinement transitions. In the asymptotic regimes of high and low temperatures, the kurtosis is essentially proportional to the quark content of the baryon number carrying effective degrees of freedom $[41,43]$; in the low-temperature phase, the effective three-quark states dominate, while at high temperatures, single quarks prevail. Therefore, at low temperatures, in the confined phase, $R_{4,2} \simeq N_{q}^{2}=9$, while in the high-temperature limit $(\mu / T \rightarrow 0)$, one recovers an ideal gas of quarks with $R_{4,2} \sim$ $1 .^{1}$ On the other hand, close to the chiral phase boundary, the kurtosis is, for the physical pion masses, strongly affected by chiral dynamics as shown below.

The properties of cumulants of the net-quark number fluctuations near the chiral phase transition were studied in LQCD [44] as well as in various models [24,26,29,33,45,46]. In particular, the significance of the quark susceptibility and the kurtosis as signatures of the deconfinement and chiral phase transitions as well as the CEP was indicated [43]. Moreover, the dependence of these fluctuations on the quark mass was analyzed in lattice QCD [47,48] and in effective models $[24,26,29,33,45]$. Nevertheless, only little is known about the dependence of the higher cumulants $c_{n}$ on the chemical potential, in particular, for $n>2$. This dependence can be computed in the PQM model, starting from the thermodynamic potential introduced in Eqs. (14) and (18). In Figs. 2-5, we show the first four cumulants obtained in the PQM model in

\footnotetext{
${ }^{1}$ More precisely, this number is $6 / \pi^{2}$, due to quantum statistics.
}

the mean-field approximation and in the FRG approach for several values of the ratio $\mu / T$. The lines of constant $\mu / T$ are indicated in the phase diagram in Fig. 1.

In Fig. 2, we show the net-quark number density normalized by $T^{3}, c_{1}=n_{q} / T^{3}$, for various values of $\mu / T$. Charge conjugation symmetry implies that the cumulant $c_{1}$, as well as all $c_{2 n+1}$, for $n=1,2,3, \ldots$, vanish at zero chemical potential $\mu=0$. At finite $\mu$, the net-quark number $n_{q}$ increases rapidly with $\mu / T$; in the chirally broken phase, the leading term is proportional to $\sinh (3 \mu / T)$. This is a direct consequence of the statistical confinement in the PQM model. For small values of Polyakov loop $l \ll 1$, the one- and two-quark modes in the partition function are suppressed, as seen in Eqs. (10) and (20). At high temperatures and chemical potentials, $n_{q}$ reduces effectively to a polynomial in $\mu / T$. In the transition region, where the chiral symmetry is partly restored, there is a change in the temperature dependence of $n_{q}$. At the pseudocritical temperature, the slope of the density is enhanced, in particular, in the mean-field calculation at larger values of $\mu / T$. In the FRG approach, the temperature dependence of $n_{q}$ is, at the corresponding value of $\mu / T$, much smoother.

The crossover chiral transition is also reflected in the isentropic trajectories. These contours of constant entropy per quark $s / n_{q}$ are followed, e.g., by a system subject to adiabatic expansion. In Fig. 1, we show isentropes labeled by $s / n_{q}$ in the PQM model obtained in the mean-field approximation and in the FRG aproach. There is a change of slope of the isentropes at the transition line, indicating a change in the equation of state. The qualitative behavior of contours of constant $s / n_{q}$ obtained in the PQM model is similar to that found in the QM model [34]. In particular, the isentropes remain smooth when the effect of long-wavelength meson fluctuations is consistently included, also in the presence of the gluon background. There is also no indication of focusing toward the CEP in the PQM model.

The influence of the finite chemical potential on the cumulant $c_{2}$ is shown in Fig. 3. At vanishing chemical potential $\mu, c_{2}$ increases monotonously with temperature, while at finite chemical potential, it develops a peak structure. The amplitude of the peak increases with increasing $\mu$ and, at the CEP, $c_{2}$ diverges. In the limit of high temperature and chemical 

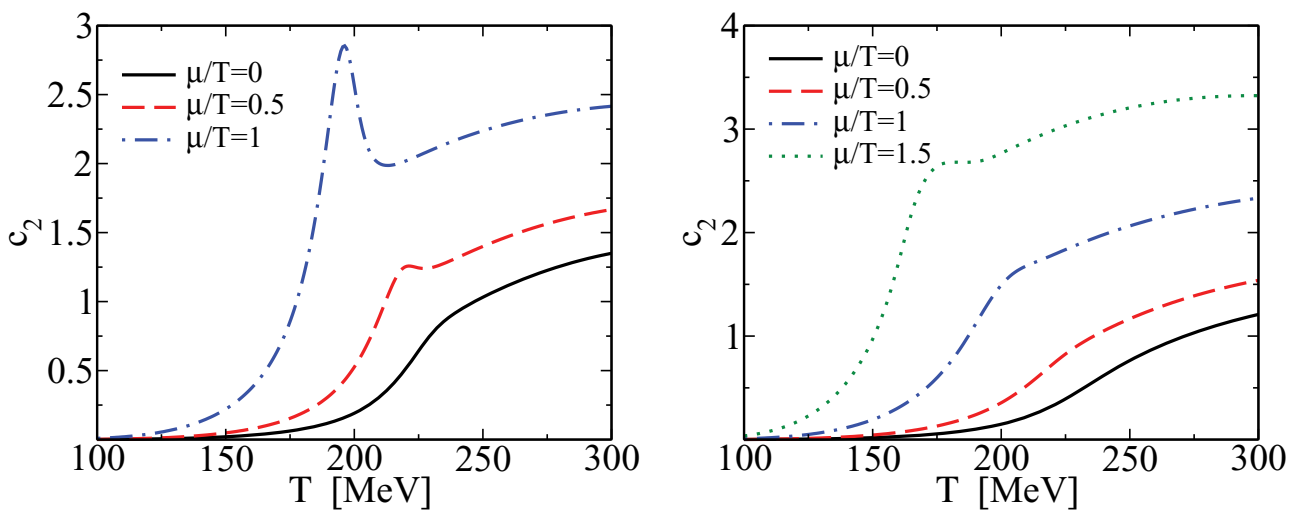

FIG. 3. (Color online) The coefficient $c_{2}$ as a function of temperature for different values of $\mu / T$ for the PQM model in the mean-field approximation (left panel) and in the FRG approach (right panel).

potential, $c_{2}$ converges to the Stefan-Boltzmann limit

$$
c_{2}^{\mathrm{SB}}=\frac{N_{c} N_{f}}{3}\left[1+\frac{3}{\pi^{2}}\left(\frac{\mu}{T}\right)^{2}\right] .
$$

As seen in Fig. 3, the peak structure in $c_{2}$ is more pronounced in the mean-field approximation than in the FRG approach, in spite of the fact that the location of the CEP in FRG is closer to, say, the $\mu / T=1$ line (see Fig. 1). Consequently, the region where the quark number fluctuations are large extends further away from the CEP in the mean-field than in the FRG approach. This result is in agreement with previous studies in the QM model, showing that the critical region shrinks due to mesonic fluctuations [23].

Thus, the cumulants $c_{1}$ and $c_{2}$ are sensitive to changes in the chemical potential and are influenced by meson fluctuations and by the gluon background. Both coefficients are, however, positive for all values of $\mu$ and $T$. This changes for higherorder cumulants; at vanishing $\mu$, the third- and fourth-order cumulants are positive or zero, while at finite $\mu / T$, both can be negative, as shown in Figs. 4 and 5. ${ }^{2}$ For large $\mu / T$, the $n=3$ and 4 cumulants exhibit a characteristic structure in the

${ }^{2}$ Cumulants of sixth and higher order are negative also at $\mu=0$ in the transition region [49].

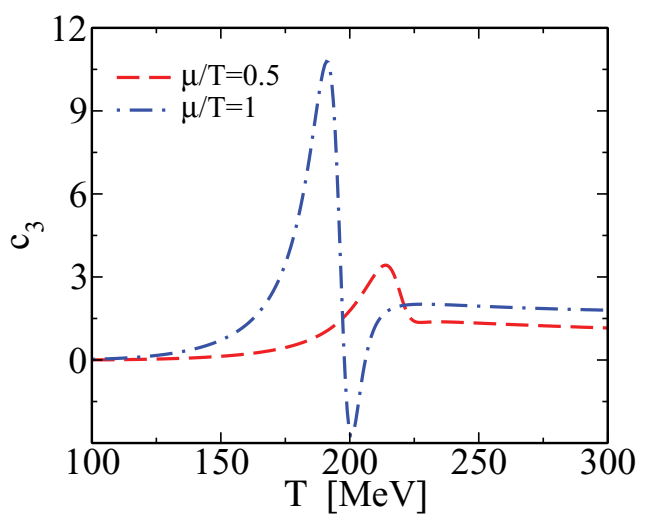

transition region; $c_{3}$ has a minimum, which can reach negative values. The amplitude of the minimum is, however, strongly suppressed by meson fluctuations, as shown in Fig. 4.

The fourth-order cumulant is negative near the crossover transition, for large values of $\mu / T$ (see Fig. 5). This implies a broadening of the net-quark number distribution, compared to Gaussian fluctuations. On the other hand, a positive $c_{4}$, as found in the broken phase, corresponds to a narrowing of the distribution. The Stefan-Boltzmann limit $c_{4}^{\mathrm{SB}}=2 N_{c} N_{f} / \pi^{2}$, which is independent of the chemical potential, is reproduced at temperatures $T \gg T_{c}$.

A comparison of the mean-field and FRG results for $c_{3}$ and $c_{4}$ shows that generalized quark susceptibilities are modified substantially by mesonic fluctuations. In the transition region, $c_{3}$ and $c_{4}$ are both suppressed in the FRG compared to the mean-field results. Hence, the critical fluctuations are important for a quantitative description of the thermodynamics near the chiral transition.

We have already indicated that both the deconfinement and chiral phase transitions are reflected in the characteristics of the kurtosis $R_{4,2}$; in the PQM model, the kurtosis drops from $R_{4,2} \simeq 9$ to $\sim 1$ in the transition region. In Figs. 6 and 7 , we show $R_{4,2}$ as a function of temperature along paths in the temperature-chemical potential plane of fixed $\mu / T$ and $s / n_{q}$, respectively. For $\mu=0$, the kurtosis exhibits a peak at

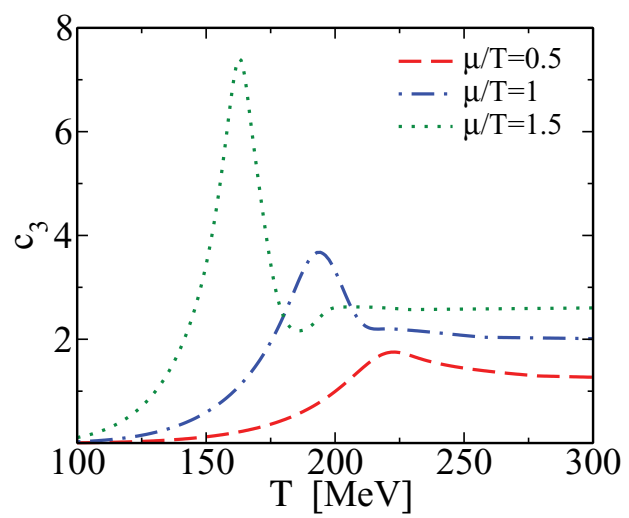

FIG. 4. (Color online) The coefficient $c_{3}$ as a function of temperature for different values of $\mu / T$ for the PQM model in the mean-field approximation (left panel) and in the FRG approach (right panel). 

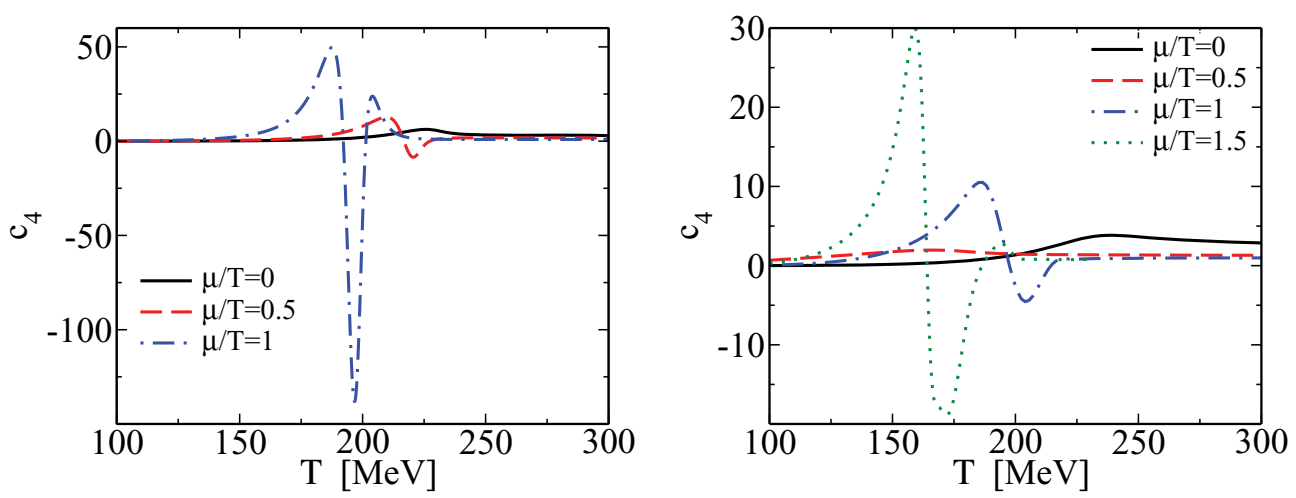

FIG. 5. (Color online) The coefficient $c_{4}$ as a function of temperature for different values of $\mu / T$ for the PQM model in the mean-field approximation (left panel) and in the FRG approach (right panel).

the transition temperature in the mean-field approximation. The height of the peak depends not only on the pion mass $[26,33,43,47]$, but also on the value of the chemical potential. The mesonic fluctuations smoothen the peak both at finite and at vanishing quark density. For nonzero $\mu$, the kurtosis turns negative, following the trend of the fourth-order cumulant.

Also, the ratio $c_{3} / c_{1}$ reflects the quark content of the baryon number carrying effective degrees of freedom, as shown in Fig. 8. At low temperatures and densities, where the thermodynamics is dominated by three-quark modes, $c_{3} / c_{1}=9$. At zero chemical potential, $c_{1}$ and $c_{3}$ both vanish but their ratio is finite. In the transition region, the ratio $c_{3} / c_{1}$ varies rapidly, much like $R_{4,2}$. At nonzero $\mu$, a peak, which grows with increasing $\mu / T$, develops below the transition. Moreover, for sufficiently large $\mu / T$, a dip appears above the transition, both in the mean-field and FRG calculations. However, in the FRG approach, the amplitude of the extrema are strongly suppressed by meson fluctuations.

\section{Scaling properties of generalized susceptibilities at nonzero chemical potential}

The general characteristics of the susceptibilities computed in the previous section can be understood by considering the critical scaling at the chiral phase transition. In the mean-field approximation, the scaling properties can be inferred from the Landau theory of phase transitions, where the singular part of the thermodynamic potential density is a polynomial in the order parameter $\sigma$,

$$
\Omega^{\text {sing }}(T, \mu ; \sigma)=\frac{1}{2} a(T, \mu) \sigma^{2}+\frac{1}{4} b(T, \mu) \sigma^{4}+\frac{1}{6} c \sigma^{6}-h \sigma .
$$

For $h=0$, there is a second-order phase transition at $a\left(T_{c}, \mu_{c}\right)=0$ if $b\left(T_{c}, \mu_{c}\right)>0$. The symmetric phase, with $\sigma=0$, is obtained for $a>0$, while the symmetry is spontaneously broken for $a<0$. For $h \neq 0$, the symmetry is explicitly broken and the transition is of the crossover type, while for $b<0$, the transition can be of first order.

For small values of the chemical potential, $\mu \ll T_{c}^{0}$ and $T \simeq T_{c}^{0}$, where $T_{c}^{0}=T_{c}(\mu=0)$ is the critical temperature of the second-order chiral phase transition at $\mu=0$, the mass term $a(T, \mu)$ is parametrized by

$$
a(T, \mu)=A \cdot\left(T-T_{c}^{0}\right)+B_{2} \mu^{2} .
$$

Here, $A>0$ and $B_{2}$ are constants. In general, the effective quartic coupling $b$ is also $T$ and $\mu$ dependent. However, this dependence is irrelevant near the critical line and far from the CEP or the tricritical point (TCP), where $a(T, \mu) \simeq 0$ and $b>0$. Therefore, in this case, we can treat $b$ as a positive constant and neglect the sixth-order term in Eq. (30), setting
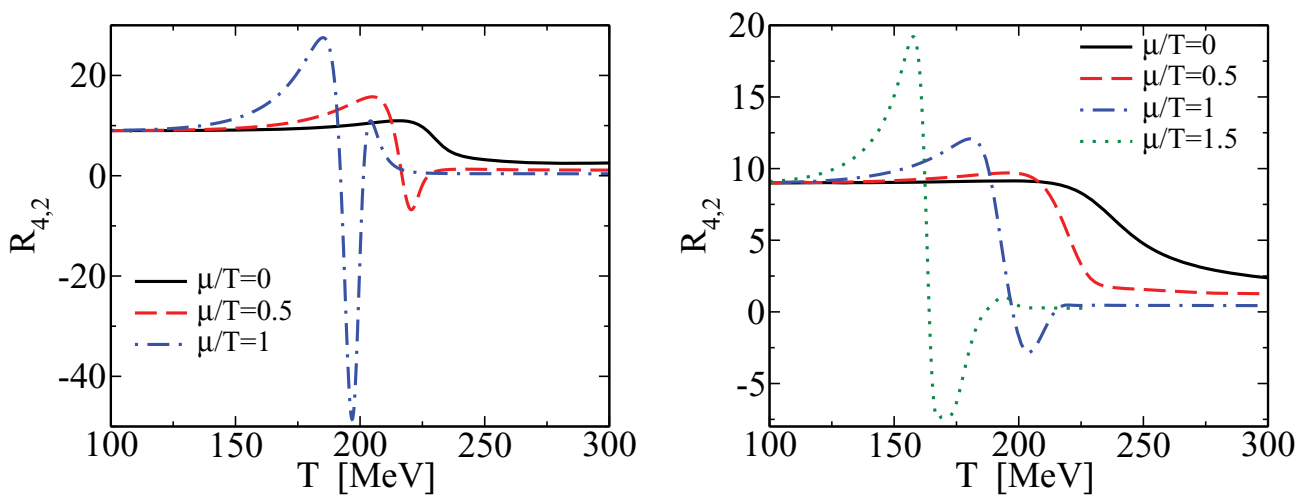

FIG. 6. (Color online) The kurtosis $R_{4,2}$ as a function of temperature for different $\mu / T$ calculated in the PQM model under the mean-field approximation (left panel) and in the FRG approach (right panel). 

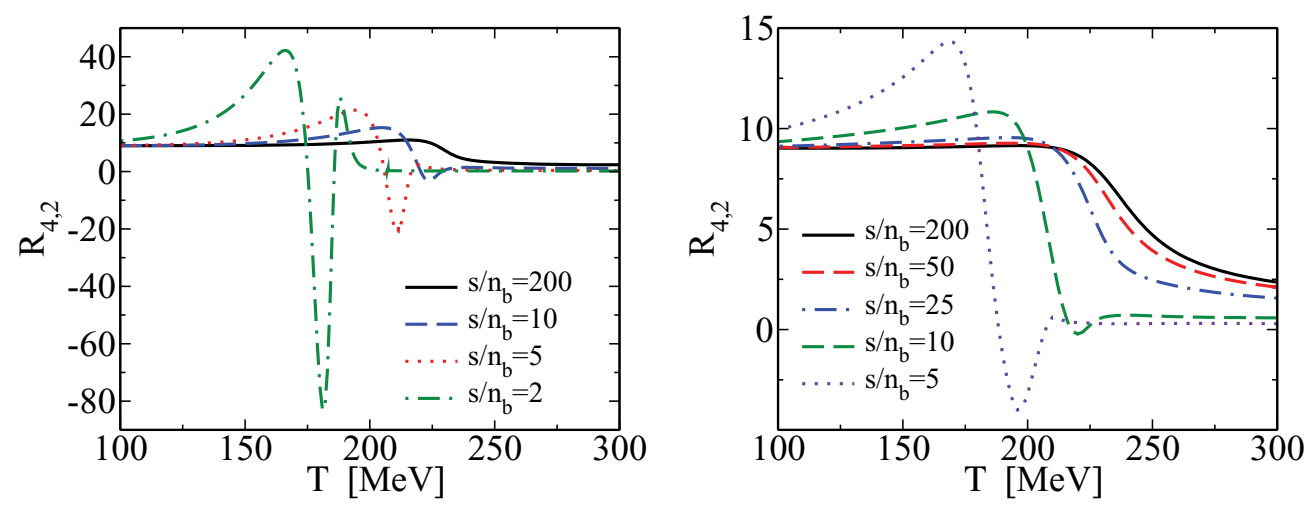

FIG. 7. (Color online) The temperature dependence of the kurtosis $R_{4,2}$ calculated in the PQM model at fixed values of the entropy density to net-quark density $\left(s / n_{q}\right)$ under the mean-field approximation (left panel) and in the FRG approach (right panel).

$c=0$. In the chiral limit $h=0$, the singular contribution to the cumulants $c_{2}$ and $c_{4}$ at $\mu=0$ are then given by

$$
\begin{aligned}
c_{2}^{\text {sing }} & =\frac{A B_{2}}{b T^{2}}\left(T-T_{c}\right) \theta\left(T_{c}-T\right), \\
c_{4}^{\text {sing }} & =\frac{6 B_{2}^{2}}{b} \theta\left(T_{c}-T\right) .
\end{aligned}
$$

Thus, $c_{2}$ is not differentiable and $c_{4}$ is discontinuous at the critical temperature. Consequently, in the chiral limit, the kurtosis $R_{4,2}$, being proportional to $c_{4}$, is discontinuous at $T_{c}$ [33].

For a finite quark mass, i.e., for $h \neq 0$, the transition is of the crossover type and the sharp structures in $c_{2}$ and $c_{4}$ are smoothened. Therefore, in the PQM model, the peaked structure in $c_{4}$ is directly linked to the value of the current quark mass and thus reflects the explicit breaking of chiral symmetry.

In the FRG approach, the critical behavior of the generalized susceptibilities, obtained in the mean-field approximation, is modified by long-wavelength meson fluctuations. Detailed studies in the QM model show that the FRG method can correctly account for long-range correlations. The resulting critical behavior is consistent with $O(4)$ scaling of the thermodynamic functions [24]. Since the gluonic background does not modify the critical dynamics related to the chiral transition, the PQM model is also expected to belong to the $O(4)$ universality class. The singular part of the thermodynamic potential is in that case controlled by the critical exponents of the three-dimensional $O(4)$-symmetric spin system. At $\mu=0$,

$$
\Omega^{\text {sing }} \sim\left(T-T_{c}\right)^{2-\alpha},
$$

which leads to the following scaling of the generalized susceptibilities:

$$
\begin{aligned}
c_{2 n}^{\text {sing }} & \sim\left(T-T_{c}\right)^{2-n-\alpha}, \\
c_{2 n+1} & =0,
\end{aligned}
$$

where $n=1,2,3, \ldots$.

In the mean-field approach, the critical exponent $\alpha=0$, while in the FRG approach, $\alpha$ is nonzero due to fluctuations. In the $O(4)$ universality class, $\alpha \simeq-0.21$, implying that fluctuations reduce the strength of the singularity. The temperature dependence of $c_{2}, c_{3}$, and $c_{4}$ is smoothened further by a nonzero quark mass.

For finite chemical potential, the coefficient $a(\mu, T)$ in the Landau potential is parametrized by

$$
a(T, \mu)=A \cdot\left(T-\bar{T}_{c}\right)+B_{1} \cdot\left(\mu-\bar{\mu}_{c}\right)+B_{2} \cdot\left(\mu-\bar{\mu}_{c}\right)^{2},
$$
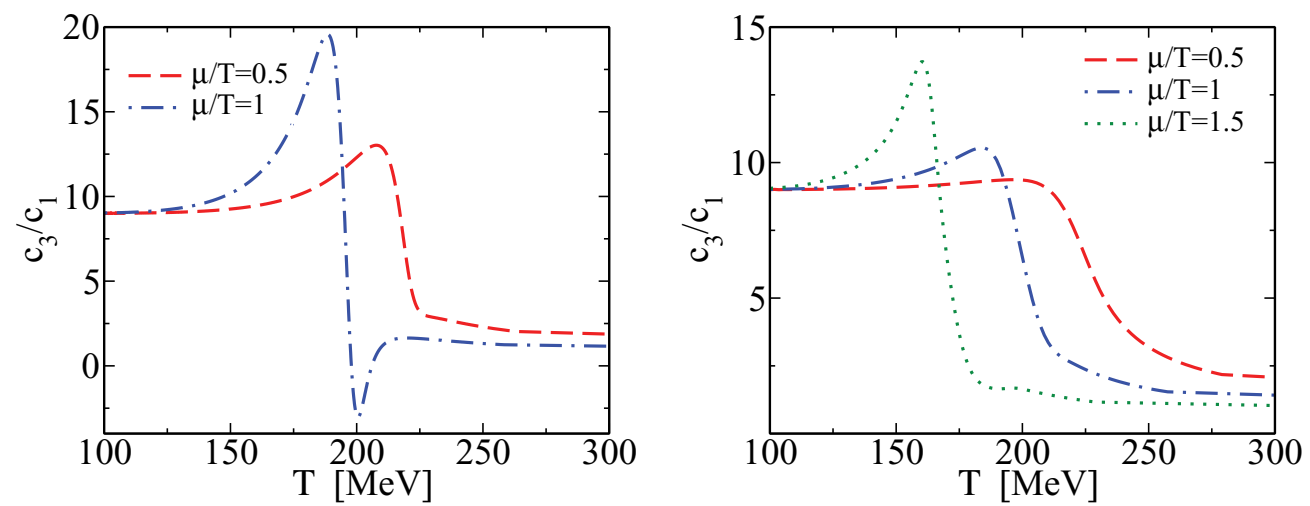

FIG. 8. (Color online) The ratio of $c_{3}$ to $c_{1}$ as a function of temperature for different values of $\mu / T$ obtained in the PQM model under the mean-field approximation (left panel) and in the FRG approach (right panel). 
where $\bar{T}_{c}$ is the critical temperature at the chemical potential $\bar{\mu}_{c}>0$. The condition $a(T, \mu)=0$ yields the equation $T_{c}(\mu)$ for the phase boundary in the neighborhood of $\left(\bar{T}_{c}, \bar{\mu}_{c}\right)$; the next-to-leading-order term, proportional to $B_{2}$, accounts for the curvature of the phase boundary. For a system which is not at the CEP or TCP, $b$ can still be treated as a positive constant, and the sixth-order term can be neglected.

In the chiral limit, the resulting mean-field expressions for the leading singular parts of the susceptibilities at $\mu \neq 0$ are

$$
\begin{aligned}
c_{1}^{\text {sing }} & =\frac{B_{1} a}{2 T^{3} b} \theta(-a), \\
c_{2}^{\text {sing }} & =\frac{B_{1}^{2}}{2 b T^{2}} \theta(-a), \\
c_{3}^{\text {sing }} & =\frac{3 B_{2} B_{1}}{b T} \theta(-a), \\
c_{4}^{\text {sing }} & =\frac{6 B_{2}^{2}}{b} \theta(-a) .
\end{aligned}
$$

Hence, $c_{2}, c_{3}$, and $c_{4}$ and, consequently, also the kurtosis, are discontinuous at the phase boundary.

In the FRG approach, the mean-field scaling at finite $\mu$ is, due to fluctuations, modified to

$$
\begin{aligned}
\Omega^{\text {sing }} & \sim(-a)^{2-\alpha}, \\
c_{n}^{\text {sing }} & \sim(-a)^{2-n-\alpha},
\end{aligned}
$$

with the $O(4)$ critical exponent $\alpha$. Since $-1<\alpha<0, c_{1}$ and $c_{2}$ remain finite, while $c_{3}, c_{4}$, and all higher-order cumulants diverge at the $O(4)$ critical line. Also, the kurtosis diverges at $T_{c}(\mu)$, owing to the singularity of $c_{4}$. By eliminating $a$ in favor of the correlation length $\xi \sim(-a)^{-\nu}$, one finds

$$
\begin{aligned}
\Omega^{\text {sing }} & \sim \xi^{(\alpha-2) / \nu}, \\
c_{n}^{\text {sing }} & \sim \xi^{(n+\alpha-2) / \nu} .
\end{aligned}
$$

The scaling properties for the net-quark number fluctuations at finite chemical potential are modified when approaching the TCP in the chiral limit or CEP at finite quark mass. At the TCP, not only $a$ in Eq. (30) vanishes, but also the coefficient $b$. In the parametrization of $a$ and $b$,

$$
a(T, \mu)=A_{a} \cdot\left(T-T_{\mathrm{TCP}}\right)+B_{a} \cdot\left(\mu-\mu_{\mathrm{TCP}}\right)
$$

and

$$
b(T, \mu)=A_{b} \cdot\left(T-T_{\mathrm{TCP}}\right)+B_{b} \cdot\left(\mu-\mu_{\mathrm{TCP}}\right),
$$

it is sufficient to retain only the leading terms. The sixth-order coupling $c>0$, as required by stability. Landau theory then yields the following expressions for the leading contributions to the generalized susceptibilities:

$$
\begin{aligned}
c_{1}^{\text {sing }} & =\frac{B_{a}}{2 T^{3}} \sqrt{\frac{-a}{c}} \theta(-a), \\
c_{n}^{\text {sing }} & =\frac{\Gamma\left(n-\frac{3}{2}\right)}{2 \Gamma\left(\frac{1}{2}\right)} \frac{(4 c)^{n-2} B_{a}^{n} T^{n-4}}{\left(b^{2}-4 a c\right)^{n-3 / 2}}, \quad n>1 .
\end{aligned}
$$

Hence, $c_{1}$ remains finite at the TCP, while the higher-order cumulants $(n \geqslant 2)$ diverge. It follows that the kurtosis also diverges, with $R_{4,2}^{\text {sing }} \sim\left(b^{2}-4 a c\right)^{-2}$.

Moreover, Eq. (49) implies that along the $a(T, \mu)=0$ line, $c_{2}^{\text {sing }}$ is, after a suitable mapping of the coordinates, inversely proportional to the distance from the TCP. By contrast, when the TCP is approached from a direction that is not tangential to the critical line, $b^{2} \ll a$ and $c_{2}^{\text {sing }}$ is inversely proportional to the square root of the transverse distance to the TCP $(\sim \sqrt{-a})[50,51]$. This implies that the critical region, defined by the properties of $c_{2}$, is elongated along the $O(4)$ critical line $[23,50,51]$.

The critical behavior near the TCP is, up to logarithmic corrections, described by mean-field theory, because the upper critical dimension equals three.

For nonvanishing external field (nonzero quark mass), the crossover transition ends in a critical endpoint (CEP). A meanfield analysis (see, also, Ref. [50]) yields the following leading singular behavior along the line defined by $a=0$ :

$$
c_{n}^{\text {sing }} \sim|v|^{2-\frac{3}{2} n}
$$

and, in any other direction,

$$
c_{n}^{\text {sing }} \sim|u|^{\frac{4}{3}-n} .
$$

The variables $v$ and $u$ introduced in Eqs. (50) and (51) are linear combinations of $\left(T-T_{\mathrm{CEP}}\right)$ and $\left(\mu-\mu_{\mathrm{CEP}}\right)$. In the $v$ direction, the kurtosis diverges scales as $R_{4,2}^{\text {sing }} \sim|v|^{-3}$, while in other directions, $R_{4,2}^{\text {sing }} \sim|u|^{-2}$.

Beyond the mean-field approximation, one expects that, along the $u=0$ line, the following scaling holds:

$$
c_{n}^{\text {sing }} \sim|v|^{-(n \beta \delta-d \nu)}
$$

and, for any other direction,

$$
c_{n}^{\text {sing }} \sim|u|^{-\left(n-2+\frac{\gamma}{\delta \beta}\right)} .
$$

Here, the critical exponents $\delta, \beta$, and $v$ are those of the threedimensional $(d=3)$ spin model, which belongs to the $Z(2)$ universality class. ${ }^{3}$ The kurtosis also diverges at the CEP, with a critical exponent that depends on the direction. Along the $u=0$ line,

$$
R_{4,2}^{\text {sing }} \sim|v|^{-2 \delta \beta},
$$

whereas, for any other direction,

$$
R_{4,2}^{\text {sing }} \sim|u|^{-2} .
$$

In terms of the correlation length $\xi$, which is proportional to $|v|^{-v}$ along the $u=0$ line and to $|u|^{-\frac{v}{\beta \delta}}$ along any other direction, we find (cf. [40])

$$
\begin{aligned}
c_{n}^{\text {sing }} & \sim \xi^{\frac{n \beta \delta}{v}-d}, \\
R_{4,2}^{\text {sing }} & \sim \xi^{2^{\frac{\beta \delta}{v}}} .
\end{aligned}
$$

We conclude that the critical structure and scaling behavior related to chiral symmetry restoration in the chiral limit are reflected in the properties of the cumulants of the net-quark number density obtained in the PQM model at finite chemical potential and pion mass.

\footnotetext{
${ }^{3}$ In the $\mathrm{Z}(2)$ universality class the $\alpha \approx 0.125, \delta \approx 5, \gamma \approx 1.25, \beta \approx$ 0.31 and $v \approx 0.64$.
} 


\section{SUMMARY AND CONCLUSIONS}

We have formulated and explored the thermodynamics of the Polyakov loop-extended quark-meson model (PQM), including mesonic fluctuations within the functional renormalization group method (FRG). The flow equations for the scale-dependent thermodynamic potential at finite temperature and density were solved in the presence of a background gluonic field.

We have shown that the nonperturbative fluctuations included in the FRG approach have an important effect on the critical properties of the system. In particular, we have focused on fluctuations of the net-quark number density and computed the first four cumulants close to the chiral transition for finite values of the chemical potential. We have indicated how the cumulants of the net-quark number density and their ratios can be used to identify the deconfinement and chiral phase transitions. We have also discussed the predictions of the Landau and scaling theories for the critical behavior of the fluctuations of the net-quark number density and the higher cumulants near the chiral phase transition.
The extension of the FRG method proposed here to account for the coupling of the quarks to the background gluon fields within the quark-meson model is of interest for unraveling the thermodynamics of QCD near the chiral phase transition at finite baryon density.

In this paper, only fluctuations of the mesonic fields are taken into account, while the effective gluon field represented by the Polyakov loops is treated on the mean-field level. Fluctuations of the Polyakov loop have been explored in model calculations [52-54]. Owing to the definition of the Polyakov loop, these studies were, explicitly or implicitly, performed in the high-temperature limit, i.e., retaining only the lowest mode. It would be interesting to find a consistent scheme where higher gluon modes also are effectively accounted for.

\section{ACKNOWLEDGMENTS}

V. Skokov acknowledges the support by the Frankfurt Institute for Advanced Studies (FIAS), and thanks M. Stephanov and C. Schmidt for valuable discussions. K. Redlich acknowledges partial support from the Polish Ministry of Science.
[1] C. R. Allton, S. Ejiri, S. J. Hands, O. Kaczmarek, F. Karsch, E. Laermann, and C. Schmidt, Phys. Rev. D 68, 014507 (2003).

[2] C. R. Allton, M. Doring, S. Ejiri, S. J. Hands, O. Kaczmarek, F. Karsch, E. Laermann, and K. Redlich, Phys. Rev. D 71, 054508 (2005).

[3] M. Cheng et al., Phys. Rev. D 77, 014511 (2008).

[4] A. Gocksch and M. Ogilvie, Phys. Rev. D 31, 877 (1985).

[5] M. Buballa, Phys. Rep. 407, 205 (2005).

[6] P. N. Meisinger and M. C. Ogilvie, Phys. Lett. B 379, 163 (1996); P. N. Meisinger, T. R. Miller, and M. C. Ogilvie, Phys. Rev. D 65, 034009 (2002).

[7] K. Fukushima, Phys. Lett. B 591, 277 (2004).

[8] F. Sannino, Phys. Rev. D 66, 034013 (2002); A. Mocsy, F. Sannino, and K. Tuominen, Phys. Rev. Lett. 92, 182302 (2004).

[9] C. Ratti, M. A. Thaler, and W. Weise, Phys. Rev. D 73, 014019 (2006).

[10] C. Sasaki, B. Friman, and K. Redlich, Phys. Rev. D 77, 034024 (2008); Phys. Rev. Lett. 99, 232301 (2007); Phys. Rev. D 75, 074013 (2007).

[11] S. Digal, E. Laermann, and H. Satz, Eur. Phys. J. C 18, 583 (2001).

[12] E. Megias, E. R. Arriola, and L. L. Salcedo, Phys. Rev. D 74, 065005 (2006).

[13] E. M. Ilgenfritz and J. Kripfganz, Z. Phys. C: Part. Fields 29, 79 (1985).

[14] K. Fukushima, Phys. Lett. B 553, 38 (2003); Phys. Rev. D 68, 045004 (2003).

[15] B. J. Schaefer, J. M. Pawlowski, and J. Wambach, Phys. Rev. D 76, 074023 (2007).

[16] E. S. Bowman and J. I. Kapusta, Phys. Rev. C 79, 015202 (2009).

[17] C. Sasaki and I. Mishustin, Phys. Rev. C 82, 035204 (2010); C. Sasaki, Acta Phys. Pol. Suppl. 3, 659 (2010); M. Harada, C. Sasaki, and S. Takemoto, Phys. Rev. D 81, 016009 (2010).

[18] T. Kahara and K. Tuominen, Phys. Rev. D 82, 114026 (2010); L. F. Palhares and E. S. Fraga, ibid. 82, 125018 (2010).
[19] C. Wetterich, Phys. Lett. B 301, 90 (1993).

[20] T. R. Morris, Int. J. Mod. Phys. A 9, 2411 (1994).

[21] U. Ellwanger, Z. Phys. C: Part. Fields 62, 503 (1994).

[22] J. Berges, N. Tetradis, and C. Wetterich, Phys. Rep. 363, 223 (2002).

[23] B. J. Schaefer and J. Wambach, Phys. Rev. D 75, 085015 (2007).

[24] B. Stokic, B. Friman, and K. Redlich, Eur. Phys. J. C 67, 425 (2010).

[25] T. K. Herbst, J. M. Pawlowski, and B.-J. Schaefer, Phys. Lett. B 696, 58 (2011).

[26] V. Skokov, B. Stokic, B. Friman, and K. Redlich, Phys. Rev. C 82, 015206 (2010).

[27] C. Ratti, S. Roessner, and W. Weise, Phys. Lett. B 649, 57 (2007).

[28] K. Fukushima, Phys. Rev. D 77, 114028 (2008); 78, 039902(E) (2008).

[29] B. J. Schaefer, M. Wagner, and J. Wambach, Phys. Rev. D 81, 074013 (2010).

[30] D. U. Jungnickel and C. Wetterich, Phys. Rev. D 53, 5142 (1996).

[31] L. F. Palhares and E. S. Fraga, Phys. Rev. D 78, 025013(E) (2008); S. Fraga, L. F. Palhares, and M. B. Pinto, ibid. 79, 065026 (2009).

[32] J. Braun, H. J. Pirner, and K. Schwenzer, Phys. Rev. D 70, 085016 (2004).

[33] V. Skokov, B. Friman, E. Nakano, K. Redlich, and B. J. Schaefer, Phys. Rev. D 82, 034029 (2010).

[34] E. Nakano, B. J. Schaefer, B. Stokic, B. Friman, and K. Redlich, Phys. Lett. B 682, 401 (2010).

[35] D. F. Litim, Nucl. Phys. B 631, 128 (2002).

[36] G. Papp et al., Phys. Rev. D 61, 096002 (2000).

[37] M. A. Stephanov, K. Rajagopal, and E. V. Shuryak, Phys. Rev. D 60, 114028 (1999).

[38] M. A. Stephanov, PoS. LAT2006, 024 (2006).

[39] C. Athanasiou, K. Rajagopal, and M. Stephanov, J. Phys. Conf. Ser. 270, 012051 (2011).

[40] M. A. Stephanov, Phys. Rev. Lett. 102, 032301 (2009).

[41] S. Ejiri, F. Karsch, and K. Redlich, Phys. Lett. B 633, 275 (2006). 
[42] F. Karsch, S. Ejiri, and K. Redlich, Nucl. Phys. A 774, 619 (2006); S. Ejiri et al., ibid. 774, 837 (2006).

[43] B. Stokic, B. Friman, and K. Redlich, Phys. Lett. B 673, 192 (2009).

[44] M. Cheng et al., Phys. Rev. D 79, 074505 (2009).

[45] W. J. Fu, Y. X. Liu, and Y. L. Wu, Phys. Rev. D 81, 014028 (2010).

[46] F. Karsch and K. Redlich, Phys. Lett. B 695, 136 (2011).

[47] F. Karsch, PoS CPOD07, 026 (2007); C. Schmidt (for RBCBielefeld Collaboration), J. Phys. G: Nucl. Part. Phys. 35, 104093 (2008).
[48] C. Miao and C. Schmidt (RBC-Bielefeld Collaboration), PoS LATTICE2008, 172 (2008).

[49] J. Wambach, B. J. Schaefer, and M. Wagner, Acta Phys. Pol. Suppl. 3, 691 (2010).

[50] Y. Hatta and T. Ikeda, Phys. Rev. D 67, 014028 (2003).

[51] C. Sasaki, B. Friman, and K. Redlich, Phys. Rev. D 75, 054026 (2007).

[52] S. Roessner, T. Hell, C. Ratti, and W. Weise, Nucl. Phys. A 814, 118 (2008).

[53] D. Smith, Phys. Rev. D 82, 034503 (2010).

[54] M. Cristoforetti, T. Hell, B. Klein, and W. Weise, Phys. Rev. D 81, 114017 (2010). 